\title{
Woody Species Richness and Diversity Following Successional Stages at Jello-Muktar Dry Afromontane Forest, South-eastern Ethiopia
}

\author{
Muktar Mohammed, Muktar Reshad, Alemayehu Beyene \\ College of Natural Resources and Environmental Science, Oda Bultum University, Chiro, Ethiopia
}

Email address:

muktarkiyyaa@gmail.com (M. Mohammed)

\section{To cite this article:}

Muktar Mohammed, Muktar Reshad, Alemayehu Beyene. Woody Species Richness and Diversity Following Successional Stages at Jello-Muktar Dry Afromontane Forest, South-eastern Ethiopia. American Journal of Agriculture and Forestry.

Vol. 7, No. 6, 2019, pp. 259-269. doi: 10.11648/j.ajaf.20190706.13

Received: August 13, 2019; Accepted: August 29, 2019; Published: October 12, 2019

\begin{abstract}
The study was carried out at $8^{0} 55^{\prime} \mathrm{N}-9^{0} 05^{\prime} \mathrm{N}$ latitude and $40^{0} 50^{\prime} \mathrm{E}-40^{0} 51^{\prime} \mathrm{E}$ longitude, at Jello-Muktar dry Afromontane forest South eastern Ethiopia to analyze changes in species composition, diversity and species richness under three successional stages. Three sites were selected each with 10 sample plots from each succssional stage. The average distance between the plots was $200 \mathrm{~m}$ and the radius was $30 \mathrm{~m}$. In each plot, identification, counting and measurement of diameter at breast height $(\mathrm{DBH})$ of all trees and shrubs $(\mathrm{DBH} \geq 10 \mathrm{~cm})$ was conducted. Comparison of richness (S) between sites and successional stages was analyzed by Chao2 estimator and Rarefaction was performed to compare species richness among sites and successional stages, for unequal number of individuals in sites. The Shannon-Wiener (H') and the Pielou indices (E) were used to assess the species diversity and evenness indices. A total of 114 species corresponding to 76 tree and 38 shrub species were recorded in the three successional stages. The MS forest was found to have the highest species richness followed by IS and ES successional stages. Analysis of Chao 2 estimator revealed that an average of $89.6 \%$ of the total species expected had been found for each successional stage. The MS sites were also found to have the highest mean H' and H'E indices. The Overall mean measure of evenness was about 0.86 which indicates that the relative homogeneity of the species in the samples was $86 \%$ of the maximum possible even population. Different conservation priorities should be implemented to maintain plant species at different successional stages.
\end{abstract}

Keywords: Richness, Successional Stages, South-eastern Ethiopia, Diversity, Dry Afro-montane

\section{Introduction}

Within an ecological community, the species composition will change over time as some species become more prominent while others may fade out of existence. As the community develops over time, vegetation grows taller, and the community becomes more established. This phenomenon was initially conceived as a natural process of ecological communities [13]. Communities with different plant composition can represent distinct mature stages in the same geographical area. The non-equilibrium hypothesis suggests that species richness should be higher at intermediate levels of disturbance [14] since conditions are not so adverse as to eliminate species. This hypothesis does not consider local physical factors such as soil and land relief and other variations associated with the species microhabitats [9, 39].

However the initial floristic composition model [20] suggests that species from late stages may also be present in the stands initial successional process [10]. Today Tropical Afro-Montane Forests are under the state of secondary succession $[17,24]$ due to anthropogenic impacts of agricultural land expansion that changed the structure and composition of these forests [3, 37, 27]. But anthropogenic disturbances can result in a higher floristic diversity, biomass and density of woody vegetation than the original mature stages [8, 31, 38] when Tropical Afro-Montane Forests are not completely transformed to pastures or fields, intermediate level.

It had been reported that most of the studies were conducted with respect to successional patterns are from the 
temperate forests and there is very little information in tropical forests [47]. Likewise, s ome early studies in JelloMuktar were focused on soil fertility and timber utilization $[34,21]$. No studies were conducted with respect to the variation in floristic composition of woody species under different succsessional patterns in Jello-Muktar forest. Indeed, there is a need to study and characterize Changes in composition and diversity of woody plants under different successional stages which could be used as a national conservation strategy for the forest biodiversity. In this study comparison of sites representing different successional stages to describe changes in florestic composition, richness and diversity of the trees and shrubs with diameter at breast height $(\mathrm{DBH}) \geq 10 \mathrm{~cm}$ under three successional stages within the Jello-Muktar forest, South-Eastern Ethiopia had been conducted.

\section{Materials and Methods}

\subsection{Study Site}

The study site is situated at $8^{0} 55^{\prime} \mathrm{N}-9^{0} 05^{\prime} \mathrm{N}$ latitude and $40^{\circ} 50^{\prime} \mathrm{E}-40^{\circ} 51^{\prime} \mathrm{E}$ longitude, about $342 \mathrm{kms}$ southeast of Addis Ababa. It covers a land area of about 1720 hectares. Its altitude ranges from 1900 to over 3310masl, having subtropical climatic condition with mean annual temperature of $10^{\circ} \mathrm{C}$ and mean rainfall of $1220 \mathrm{~mm}$. Its length of growing period ranges from 210-270days [33].

It is part of the Chercher highlands in Harerghe with extensive mountain range separating the Rift system from the Eastern plateau and lowlands, having numerous microcatchments with diversified bio-physical and socioeconomic environments [36]. Anthropogenic processes due to shifting cultivation and forest clearing has led to continuous removal of soil materials that strongly affected the micro-climate and soil development of the area [21]. The study area is dissected by streams due to their erosive processes for prolonged period of time. The sub-watershed is within Wabi-shebele and Rift Valley drainage systems, drained by Chiro Qela and Jello perennial streams towards the Rift system while perennial streams like Welenso and Ula Quni and seasonal streams like Rukele Arba Feno and Dingete draining towards Wabi Shebelle drainage system [19].

\subsection{Selection of Sites}

Nine sites represented by three successional stages were selected as follows [4]: a) 3 disturbed sites from early stage (ES) of succession about 15 years which consisted of secondary vegetation; b), 3 sites from Intermediate stage (IS) of succession representing a transition between early and mature forests of 30 years and c), 3 sites from Mature stage (MS) succession with relatively well preserved sites of 45 years, characterized by the typical structure and cover of mature forest. Information about the time since last major disturbance (i.e., cutting, clearing and farming) obtained from interviews with key informants was used for the selection of the secondary vegetation sites [4].

\subsection{Plot Lay Out and Vegetation Sampling}

A total of 90 sample plots were used for the three successional stages of the forest. Ten sample plots each with radius of $30 \mathrm{~m}$ were laid in each site for each successional stage. The average distance between the plots was $200 \mathrm{~m}$. In each of the plots, diameter at breast height (DBH) of all trees and shrubs $(\mathrm{DBH} \geq 10 \mathrm{~cm})$ was measured with diameter tape. Identification of woody plant species in each of the sample plot was conducted with the help of para-taxonomists and species difficult for identification in the field were pressed and taken to the National Herbarium, Addis Ababa University. Vernacular names of the plant species were recorded in the field. The nomenclature of the taxa followed Flora of Ethiopia and Eritrea (FEE).

\subsection{Data Analysis}

The number of individuals for each species was counted and the Shannon-Wiener $\left(H^{\prime}\right)$ and the Pielou indices $(E)$ were used to assess the species diversity and evenness as:

\subsubsection{Species Diversity}

The Shannon-Weaver's index $\left(\mathrm{H}^{\prime}\right)$ was used as measure of diversity and calculated as $\mathrm{H}^{1}=-\Sigma P i \ln P i$ where: $i$, is the proportion of the species relative to the total number of species (pi) and $\ln$ is a natural logarithm.

\subsubsection{Species Richness (S)}

Species richness as the number of species present in an ecosystem was calculated as: $\mathrm{S}=\mathrm{Sn}$ Where: $n$ is number of species.

\subsubsection{Species Evenness $\left(\mathrm{H}^{\prime} \mathrm{E}\right)$}

Species evenness $\mathrm{H}^{\prime} \mathrm{E}$ is the proportion of individuals among species in an ecosystem and it is assessed by Shannon's equitability index and calculated as: $H^{\prime} E=H^{\prime} / H$ max Where: $\mathrm{H}_{\max }$ is defined as $\ln S$

An incidence-based species richness Chao 2 estimator based on the number of unique, and the number of duplicates was used to compare richness (S) between sites and successional stages [12]. Rarefaction analysis as performed to compare species richness among sites and successional stages for samples with different number of individuals [41]. The analysis was conducted by Estimate $\mathrm{S}$ v. 8.0 [11]. Since Shannon index is approximately normally distributed, making comparison with parametric tests is feasible and independent t-test was performed to evaluate the differences.

\section{Results and Discussion}

\subsection{Floristic Composition and Species Richness}

A total of 114 species (76 tree and 38 shrub species) corresponding to 51 families were recorded in the three successional stages (Tables 1-3). The number of species 
richness recorded in this study of which $66.7 \%$ were trees is comparable to the numbers reported for other similar forest types [30, 32, 28] but higher than the total number of species recorded at Ades dry afromontane forest of the same agroecology [34] and elsewhere in Ethiopia for studies from similar vegetation types $[45,29,42,1,2]$. It is also higher than the number of species reported from tropical dry forest in southwest Mexico [4] and in the temperate landscape of southern Chile [15]. This may be due to the presence of participatory forest management at Jello-Muktar forest. However, it is lower than the number of species reported for Senka meda with 139 species [43].

The family with the highest number of species were Fabaceae and Myrtaceae each with 7 species followed by Rosaceae (6), Myrsinaceae (5), Euphorbiaceae (5), Rutaceae (5), Flacourtiaceae (4), Oleaceae (4), Celastraceae (4), Papilionoideae (4), Verbenaceae (4), Apocynaceae (3), Araliaceae (3), Asteraceae (3), Flacourtiaceae (3), Moraceae (3), and Rubiaceae (3). These families represented $60.2 \%$ of the total species in the study site while the remaining families had 2 or 1 species and represented only $39.8 \%$ of the total species (Tables 1-3). The dominance of Fabaceae and Myrtaceae in terms of the number of species is in line with some studies reported for forests of other Tropical areas [22, $23,4]$.

There also existed variation between successional stages in terms of the floristic composition of woody species at the family level. Twelve families namely: Asteraceae,
Cupressaceae, Myrsinaceae, Rosaceae, Sterculiaceae, Myrsinaceae, Sapindaceae, Sapotaceae, Flacourtiaceae, Hypericaceae, Fabaceae and Euphorbiaceae were recorded in all successional stages while the families of Balanitaceae, Bambusaceae, Bignoniaceae, Ebenaceae, Loganiaceae, Phytolacaceae, Pittosporaceae, Podocarpaceae, Sapindaceae and Vitaceae were only found in one of the three successional stages (Tables 1-3).

There were 28 families in the ES successional stage (Table 1). In this succsseeional stage, 16 families were each represented by one species only while the rest were represented by at least two species. This may be due dispersal strategies of the species that can affect its germination according to some studies from tropical dry forests in ES where the seed bank is severely depleted or absent $[25,40]$. In addition to the dispersal strategies of the species, other studies also reported that soil conditions, topography and microclimate of the forests can affect the species composition and succession dynamics $[46,26,7,16$, $24,4]$.

All the three sites of ES were dominated by species of Vernonia amygdalina and Vernonia auriculifera. Such species which are dominant in the ES sites are indicative of secondary succession and have a high capacity to colonize degraded areas resulting in distinct microclimatic changes and low water retention that could hamper the germination and establishment of typical of mature forests species [24, 4].

Table 1. Plant species Composition (DBH $\geq 10 \mathrm{~cm})$ identified in three sites under ES successional stage at Jello-Muktar dry afromontane forest, South-Eastern Ethiopia.

\begin{tabular}{|c|c|c|c|c|c|}
\hline \multirow{3}{*}{ Family } & \multirow{3}{*}{ Species } & \multirow{3}{*}{ Life form } & \multicolumn{3}{|c|}{ Successional Stages } \\
\hline & & & \multicolumn{3}{|c|}{$E S$} \\
\hline & & & 1 & 2 & 3 \\
\hline Agavaceae & Dracaena steudneri & Shrub & $\mathrm{x}$ & & \\
\hline Anacardiaceae & Schinus molle & Tree & $\mathrm{x}$ & & \\
\hline \multirow{2}{*}{ Apocynaceae } & Acokanthera schimperi & Tree & & $\mathrm{x}$ & \\
\hline & Carissa edulis & Shrub & & $\mathrm{x}$ & $\mathrm{x}$ \\
\hline \multirow{2}{*}{ Araliaceae } & Cussonia holstii & Tree & & $\mathrm{x}$ & \\
\hline & Schefflera abyssinica & Tree & & & $\mathrm{x}$ \\
\hline \multirow{2}{*}{ Asteraceae } & Vernonia amygdalina & Tree & $\mathrm{x}$ & $\mathrm{X}$ & $\mathrm{x}$ \\
\hline & Vernonia auriculifera & Shrub & $\mathrm{x}$ & & $\mathrm{x}$ \\
\hline Balanitaceae & Balanytus aegyptica & Tree & $\mathrm{x}$ & & \\
\hline \multirow{2}{*}{ Boraginaceae } & Ehretia cymosa & Tree & & & $\mathrm{x}$ \\
\hline & Cordia africana & Tree & & $\mathrm{x}$ & $\mathrm{x}$ \\
\hline \multirow{2}{*}{ Cupressaceae } & Cupressus lusitanica & Tree & & & $\mathrm{x}$ \\
\hline & Juniperus procera & Tree & $\mathrm{x}$ & $\mathrm{x}$ & $\mathrm{x}$ \\
\hline \multirow{2}{*}{ Euphorbiaceae } & Croton macrostachys & Tree & & $\mathrm{x}$ & \\
\hline & Macaranga kilimandsharica & Tree & $\mathrm{x}$ & $\mathrm{x}$ & \\
\hline \multirow{6}{*}{ Fabaceae } & Acacia decurrence & Tree & & & $\mathrm{x}$ \\
\hline & Albizzia gummifera & Tree & & & $\mathrm{x}$ \\
\hline & Calpunia subdecandra & Shrub & & & $\mathrm{x}$ \\
\hline & Calpurina aura & Tree & & $\mathrm{x}$ & \\
\hline & Leucena leucocephala & Tree & & & $\mathrm{x}$ \\
\hline & Dovyalis abyssinica & Shrub & $\mathrm{x}$ & $\mathrm{x}$ & \\
\hline Icacinaceae & Apodytes dimidiata & Shrub & & $\mathrm{x}$ & \\
\hline Lamiacae & Ocimum lamifolium & Shrub & & $\mathrm{x}$ & \\
\hline Meliaceae & Lepidotrichilia volkensis & Tree & & $\mathrm{x}$ & \\
\hline \multirow{2}{*}{ Moraceae } & Ficus sur & Shrub & & $\mathrm{x}$ & \\
\hline & Ficus thonningi & Tree & $\mathrm{x}$ & & \\
\hline Myrsinaceae & Myrsine africana & Shrub & & $\mathrm{x}$ & \\
\hline
\end{tabular}




\begin{tabular}{|c|c|c|c|c|c|}
\hline \multirow{3}{*}{ Family } & \multirow{3}{*}{ Species } & \multirow{3}{*}{ Life form } & \multicolumn{3}{|c|}{ Successional Stages } \\
\hline & & & \multicolumn{3}{|c|}{$E S$} \\
\hline & & & 1 & 2 & 3 \\
\hline \multirow{2}{*}{ Myrtaceae } & Eucalyptus saligna & Tree & & $\mathrm{x}$ & \\
\hline & Psidium guajava & Tree & & $\mathrm{x}$ & \\
\hline Oleaceae & Olea capensis & Tree & $\mathrm{x}$ & $\mathrm{x}$ & $\mathrm{x}$ \\
\hline \multirow{2}{*}{ Papilionoideae } & Erythrina abyssinica & Shrub & $\mathrm{x}$ & $\mathrm{x}$ & \\
\hline & Erythrina brucei & Tree & & & $x$ \\
\hline Passifloraceae & Passiflora edulis & Tree & & & $\mathrm{x}$ \\
\hline \multirow{2}{*}{ Pittosporaceae } & Pittosporum abyssinicum & Tree & $\mathrm{x}$ & & \\
\hline & Rhamnus prenoids & Tree & $\mathrm{x}$ & $\mathrm{x}$ & \\
\hline \multirow{3}{*}{ Rhamnaceae } & Hagenia abyssinica & Shrub & $\mathrm{x}$ & & \\
\hline & Prunus africana & Tree & & & $\mathrm{x}$ \\
\hline & Rosa abyssinica & Tree & $\mathrm{x}$ & $\mathrm{x}$ & $\mathrm{x}$ \\
\hline Rutaceae & Vepris dainellii & Tree & $\mathrm{x}$ & & \\
\hline Sapotaceae & Aningeria altissima & Tree & $\mathrm{x}$ & $\mathrm{x}$ & \\
\hline Simaroubaceae & Brucea antidysenterica & Tree & & & $\mathrm{x}$ \\
\hline Solanaceae & Discopodium penninervium & Shrub & & & $\mathrm{x}$ \\
\hline Sterculiaceae & Dombeya torrida & Shrub & & $\mathrm{x}$ & \\
\hline Ulmaceae & Celtis africana & Tree & & & $\mathrm{x}$ \\
\hline \multirow{2}{*}{ Verbenaceae } & Clerodendron myricoides & Tree & $\mathrm{x}$ & $\mathrm{x}$ & \\
\hline & Premna resinosa & Shrub & & & $\mathrm{x}$ \\
\hline
\end{tabular}

Species of Juniperus procera, Rosa abyssinica, Olea capensis, Vernonia amygdalina, Vernonia auriculifera, Crotonon macrostachys, Apodytus diminiata and Prunus africana were recorded in all of the successional stages while species of Cussonia holstii, Balanytus aegyptica, Caesalpina decapetala, Delonix regia, Vitis vinifera, Euphorbia abyssinica, Leucena leucocephala, Strychnos mitis, Ekebergia capensis, Morus alba, Eucalyptus camaldulensis, Sizygeem guineense, Gardenia lutea, Fagaropsis angolensis, Vepris dainellii, Teclea nobilis, Lippia adeonsis, and Euphorbia pulcherrima were observed only in one of the three successional stages.
A total of 35 families were recorded in IS stage (Table 2) which accounts about $57 \%$ of the total number of woody species identified in this study. In IS successional stage the family of Proteaceae comprise the largest number of species (6) followed by Myrtaceae (5) and Oleaceae (4). Species of Vernonia amygdalina, Vernonia auriculifera, Cupressus lusitanica, Juniperus procera, Croton macrostachys, Acacia decurrence, Apodytes dimidiate, Ocimum lamifolium, Ficus sur, Myrsine aricana, Pinus patula, Rumex abyssinicus, Prunus africana and Rosa abyssinica were observed in all the three sites of IS (Table 2).

Table 2. Plant species Composition (DBH $\geq 10 \mathrm{~cm}$ ) identified in 3 sites under IS successional stage at Jello-Muktar dry afromontane forest, South-Eastern Ethiopia.

\begin{tabular}{|c|c|c|c|c|c|}
\hline \multirow{3}{*}{ Family } & \multirow{3}{*}{ Species } & \multirow{3}{*}{ Life form } & \multicolumn{3}{|c|}{ Successional Stages } \\
\hline & & & \multicolumn{3}{|c|}{$I S$} \\
\hline & & & 1 & 2 & 3 \\
\hline Apocynaceae & Carissa spinarum & Shrub & & $\mathrm{X}$ & \\
\hline Aquifoliaceae & Ilex mitis & Tree & $\mathrm{X}$ & & \\
\hline \multirow{3}{*}{ Araliaceae } & Polyscias fulva & Tree & & & $\mathrm{X}$ \\
\hline & Schefflera abyssinica & Tree & & & $X$ \\
\hline & Vernonia amygdalina & Tree & $\mathrm{X}$ & $\mathrm{X}$ & $\mathrm{X}$ \\
\hline \multirow[t]{2}{*}{ Asteraceae } & Vernonia auriculifera & Shrub & $\mathrm{X}$ & $\mathrm{X}$ & $X$ \\
\hline & Vernonia leopoldii & Shrub & & $\mathrm{X}$ & $\mathrm{X}$ \\
\hline \multirow[t]{2}{*}{ Boraginaceae } & Ehretia cymosa & Tree & & $\mathrm{X}$ & $\mathrm{X}$ \\
\hline & Chata edulis & Tree & & $\mathrm{X}$ & \\
\hline \multirow{2}{*}{ Celastraceae } & Maytenus arbutifolia & Tree & & & $X$ \\
\hline & Maytenus obscura & Tree & & & $\mathrm{X}$ \\
\hline \multirow{2}{*}{ Cupressaceae } & Cupressus lusitanica & Tree & $\mathrm{X}$ & $\mathrm{X}$ & $X$ \\
\hline & Juniperus procera & Tree & X & $X$ & X \\
\hline Ebenaceae & Diospyros abyssinica & Tree & & & \\
\hline Ericaeae & Erica arborea & Shrub & & $\mathrm{X}$ & \\
\hline \multirow{2}{*}{ Euphorbiaceae } & Croton macrostachys & Tree & $\mathrm{X}$ & $\mathrm{X}$ & $\mathrm{X}$ \\
\hline & Macaranga kilimandsharica & Tree & & & $\mathrm{x}$ \\
\hline \multirow{4}{*}{ Fabaceae } & Acacia decurrence & Tree & $\mathrm{x}$ & $\mathrm{x}$ & $\mathrm{x}$ \\
\hline & Calpunia subdecandra & Shrub & $\mathrm{x}$ & & \\
\hline & Calpurina aura & Tree & & $\mathrm{x}$ & \\
\hline & Dovyalis abyssinica & Shrub & $\mathrm{x}$ & & \\
\hline \multirow{2}{*}{ Flacourtiaceae } & Dovyalis vericosa & Shrub & & $\mathrm{x}$ & $\mathrm{x}$ \\
\hline & Flacourtia indica & Shrub & $\mathrm{x}$ & & \\
\hline Icacinaceae & Apodytes dimidiata & Shrub & $\mathrm{x}$ & $\mathrm{x}$ & $\mathrm{x}$ \\
\hline
\end{tabular}




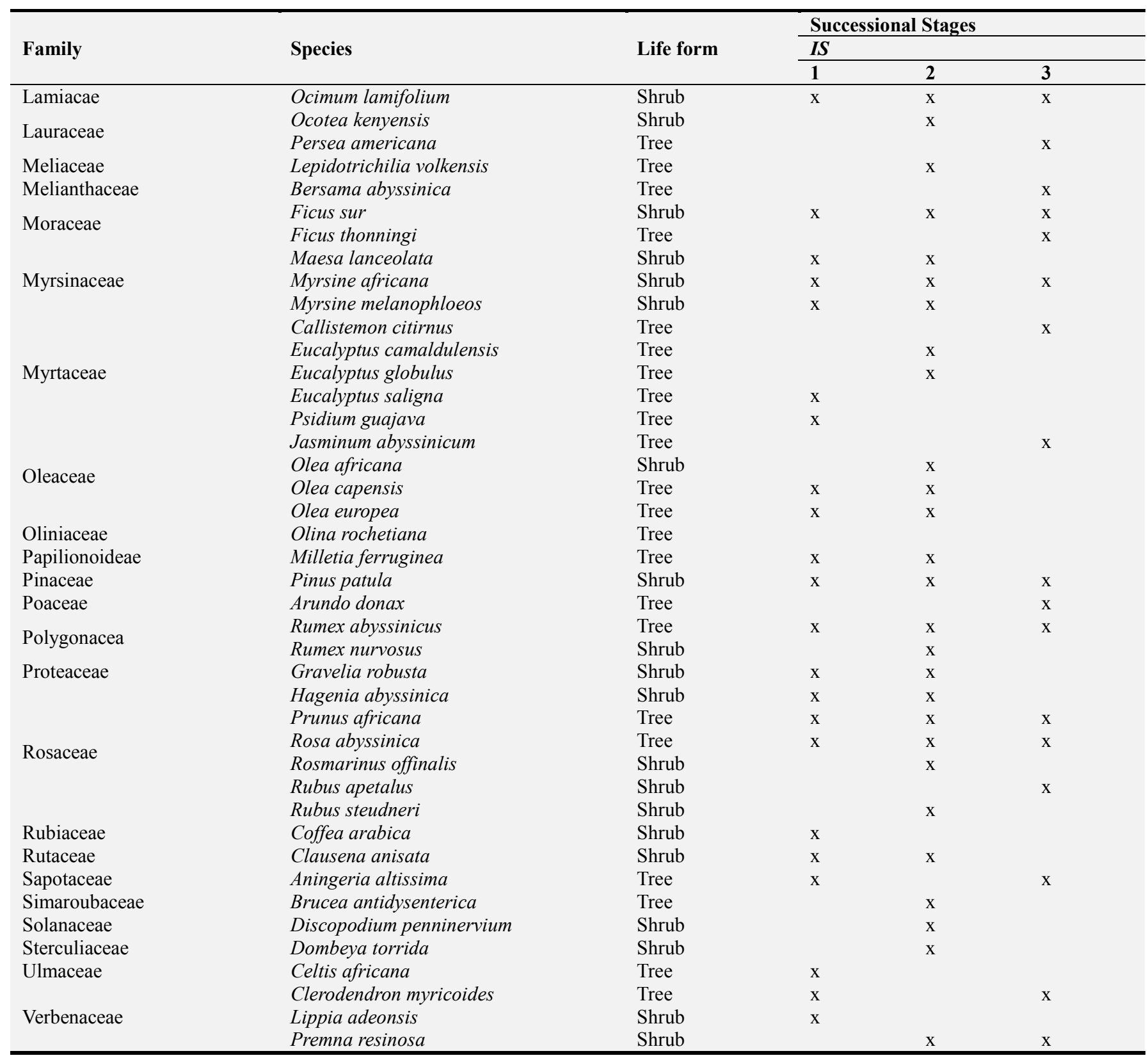

The MS successional stages had the highest number of families, 47 families comprising $77.2 \%$ of the total number of species in this study (Table 3).

Table 3. Plant species Composition (DBH $\geq 10 \mathrm{~cm})$ identified in 3 sites under MS successional stage at Jello-Muktar dry afromontane, South-Eastern Ethiopia.

\begin{tabular}{|c|c|c|c|c|c|}
\hline \multirow{3}{*}{ Family } & \multirow{3}{*}{ Species } & \multirow{3}{*}{ Life form } & \multicolumn{3}{|c|}{ Successional Stages } \\
\hline & & & \multicolumn{3}{|c|}{$M S$} \\
\hline & & & 1 & 2 & 3 \\
\hline Agavaceae & Dracaena steudneri & Shrub & & & $\mathrm{x}$ \\
\hline Anacardiaceae & Rhus glutinosa & Shrub & & $\mathrm{x}$ & $\mathrm{x}$ \\
\hline \multirow{2}{*}{ Apocynaceae } & Acokanthera schimperi & Tree & $\mathrm{x}$ & $\mathrm{x}$ & \\
\hline & Carissa spinarum & Shrub & $\mathrm{x}$ & & \\
\hline Aquifoliaceae & Ilex mitis & Tree & $\mathrm{x}$ & & \\
\hline \multirow{2}{*}{ Araliaceae } & Polyscias fulva & Tree & & $\mathrm{x}$ & $\mathrm{x}$ \\
\hline & Schefflera abyssinica & Tree & $\mathrm{x}$ & $\mathrm{x}$ & $\mathrm{x}$ \\
\hline \multirow{3}{*}{ Asteraceae } & Vernonia amygdalina & Tree & & $\mathrm{x}$ & $\mathrm{x}$ \\
\hline & Vernonia auriculifera & Shrub & & $\mathrm{x}$ & \\
\hline & Vernonia leopoldii & Shrub & & $\mathrm{x}$ & \\
\hline Bambusaceae & Arundinaria alpina & Tree & $\mathrm{x}$ & $\mathrm{x}$ & $\mathrm{x}$ \\
\hline Bignoniaceae & Jacaranda mimosifolia & Tree & & & $\mathrm{x}$ \\
\hline
\end{tabular}




\begin{tabular}{|c|c|c|c|c|c|}
\hline \multirow{3}{*}{ Family } & \multirow{3}{*}{ Species } & \multirow{3}{*}{ Life form } & \multicolumn{3}{|c|}{ Successional Stages } \\
\hline & & & \multicolumn{3}{|c|}{$M S$} \\
\hline & & & 1 & 2 & 3 \\
\hline \multirow{2}{*}{ Boraginaceae } & Ehretia cymosa & Tree & & $\mathrm{x}$ & \\
\hline & Cordia africana & Tree & & $\mathrm{x}$ & $\mathrm{x}$ \\
\hline \multirow{3}{*}{ Caesalpinioideae } & Caesalpina decapetala & Tree & & $\mathrm{x}$ & \\
\hline & Delonix regia & Tree & & & $\mathrm{x}$ \\
\hline & Chata edulis & Tree & $\mathrm{x}$ & & \\
\hline \multirow{3}{*}{ Celastraceae } & Maytenus addat & Tree & $\mathrm{x}$ & $\mathrm{x}$ & \\
\hline & Maytenus arbutifolia & Tree & & $\mathrm{x}$ & $\mathrm{x}$ \\
\hline & Maytenus obscura & Tree & $\mathrm{x}$ & & $\mathrm{x}$ \\
\hline \multirow{2}{*}{ Cupressaceae } & Cupressus lusitanica & Tree & & & \\
\hline & Juniperus procera & Tree & $\mathrm{x}$ & $\mathrm{x}$ & $\mathrm{x}$ \\
\hline Ebenaceae & Diospyros abyssinica & Tree & $\mathrm{x}$ & & $\mathrm{x}$ \\
\hline \multirow[t]{2}{*}{ Ericaeae } & Erica arborea & Shrub & $\mathrm{x}$ & & $\mathrm{x}$ \\
\hline & Croton macrostachys & Tree & $\mathrm{x}$ & $\mathrm{x}$ & $\mathrm{x}$ \\
\hline \multirow{5}{*}{ Euphorbiaceae } & Euphorbia abyssinica & Tree & & & $\mathrm{x}$ \\
\hline & Euphorbia pulcherrima & Tree & & & $\mathrm{x}$ \\
\hline & Macaranga kilimandsharica & Tree & & $\mathrm{x}$ & \\
\hline & Sapium ellipticum & Tree & $\mathrm{x}$ & $\mathrm{x}$ & \\
\hline & Albizzia gummifera & Tree & & $\mathrm{x}$ & $\mathrm{x}$ \\
\hline \multirow{3}{*}{ Fabaceae } & Calpurina aura & Tree & & $\mathrm{x}$ & \\
\hline & Entada abyssinica & Shrub & & $\mathrm{x}$ & \\
\hline & Dovyalis abyssinica & Shrub & $\mathrm{x}$ & & \\
\hline Hypericaceae & Hypericum revoltum & Tree & $\mathrm{x}$ & $\mathrm{x}$ & \\
\hline Icacinaceae & Apodytes dimidiata & Shrub & $\mathrm{x}$ & $\mathrm{x}$ & \\
\hline Lamiacae & Ocimum lamifolium & Shrub & $\mathrm{x}$ & & \\
\hline Lauraceae & Persea americana & Tree & $\mathrm{x}$ & & $\mathrm{x}$ \\
\hline \multirow{2}{*}{ Loganiaceae } & Buddleja polystacha & Tree & & $\mathrm{x}$ & \\
\hline & Strychnos mitis & Tree & & $\mathrm{x}$ & \\
\hline & Ekebergia capensis & Tree & & $\mathrm{x}$ & \\
\hline Meliaceae & Lepidotrichilia volkensis & Tree & $\mathrm{x}$ & & $\mathrm{x}$ \\
\hline Melianthaceae & Bersama abyssinica & Tree & $\mathrm{x}$ & $\mathrm{x}$ & $\mathrm{x}$ \\
\hline Moracene & Ficus sur & Shrub & $\mathrm{x}$ & & \\
\hline Moraceae & Morus alba & Tree & & & $\mathrm{x}$ \\
\hline & Embelia schimpri & Tree & & $\mathrm{x}$ & \\
\hline Myrcinaceae & Myrsine africana & Shrub & $\mathrm{x}$ & $\mathrm{x}$ & \\
\hline Myrsinaceae & Myrsine melanophloeos & Shrub & $\mathrm{x}$ & $\mathrm{x}$ & $\mathrm{x}$ \\
\hline & Rapanea simensis & Tree & $\mathrm{x}$ & $\mathrm{x}$ & $\mathrm{x}$ \\
\hline & Callistemon citirnus & Tree & $\mathrm{x}$ & & $\mathrm{x}$ \\
\hline & Casuarina equisetifolia & Tree & & & $\mathrm{x}$ \\
\hline Myrtaceae & Psidium guajava & Tree & & & $\mathrm{x}$ \\
\hline & Sizygeem guineense & Tree & & & $\mathrm{x}$ \\
\hline & Jasminum abyssinicum & Tree & $\mathrm{x}$ & $\mathrm{x}$ & $\mathrm{x}$ \\
\hline & Olea africana & Shrub & & $\mathrm{x}$ & \\
\hline Oleaceae & Olea capensis & Tree & & $\mathrm{x}$ & $\mathrm{x}$ \\
\hline & Olea europea & Tree & $\mathrm{x}$ & $\mathrm{x}$ & \\
\hline Oliniaceae & Olina rochetiana & Tree & & & $\mathrm{x}$ \\
\hline & Milletia ferruginea & Tree & $\mathrm{x}$ & & \\
\hline Papilionoideae & Erythrina brucei & Tree & & & $\mathrm{x}$ \\
\hline Passifloraceae & Passiflora edulis & Tree & $\mathrm{x}$ & & \\
\hline Phytolacaceae & Phytolaca deodecandra & Tree & & $\mathrm{x}$ & \\
\hline & Pinus patula & Shrub & & $\mathrm{x}$ & $\mathrm{x}$ \\
\hline Pinaceae & Pinus radiata & Tree & & $\mathrm{x}$ & \\
\hline Poaceae & Arundo donax & Tree & $\mathrm{x}$ & & $\mathrm{x}$ \\
\hline Podocarpaceae & Podocarpus falcatus & Tree & & $\mathrm{x}$ & \\
\hline & Rumex abyssinicus & Tree & $\mathrm{x}$ & $\mathrm{x}$ & $\mathrm{x}$ \\
\hline Polygonacea & Rumex nurvosus & Shrub & & & $\mathrm{x}$ \\
\hline Proteaceae & Gravelia robusta & Shrub & & & $\mathrm{x}$ \\
\hline & Hagenia abyssinica & Shrub & & $\mathrm{x}$ & \\
\hline & Prunus africana & Tree & $\mathrm{x}$ & $\mathrm{x}$ & $\mathrm{x}$ \\
\hline Recacene & Rosa abyssinica & Tree & $\mathrm{x}$ & $\mathrm{x}$ & $\mathrm{x}$ \\
\hline Rosaceae & Rosmarinus offinalis & Shrub & & $\mathrm{x}$ & $\mathrm{x}$ \\
\hline & Rubus apetalus & Shrub & $\mathrm{x}$ & $\mathrm{x}$ & \\
\hline & Rubus steudneri & Shrub & & $\mathrm{x}$ & \\
\hline Rubiaceae & Coffea arabica & Shrub & & $\mathrm{x}$ & \\
\hline
\end{tabular}




\begin{tabular}{|c|c|c|c|c|c|}
\hline \multirow{3}{*}{ Family } & \multirow{3}{*}{ Species } & \multirow{3}{*}{ Life form } & \multicolumn{3}{|c|}{ Successional Stages } \\
\hline & & & \multicolumn{3}{|c|}{$M S$} \\
\hline & & & 1 & 2 & 3 \\
\hline \multirow{6}{*}{ Rutaceae } & Gardenia lutea & Tree & & & $\mathrm{X}$ \\
\hline & Rytigynia neglecta & Tree & & $\mathrm{x}$ & \\
\hline & Clausena anisata & Shrub & $\mathrm{x}$ & & $\mathrm{X}$ \\
\hline & Diphasia dainelli & Tree & $\mathrm{x}$ & & \\
\hline & Fagaropsis angolensis & Tree & & & $\mathrm{X}$ \\
\hline & Teclea nobilis & Tree & $\mathrm{x}$ & & \\
\hline Sapindaceae & Dodonaea angustifolia & Tree & $\mathrm{x}$ & & \\
\hline \multirow{2}{*}{ Sapotaceae } & Aningeria altissima & Tree & & $\mathrm{x}$ & \\
\hline & Mimusops kummel & Tree & & $\mathrm{x}$ & $\mathrm{x}$ \\
\hline Simaroubaceae & Brucea antidysenterica & Tree & $\mathrm{x}$ & & $\mathrm{x}$ \\
\hline Solanaceae & Discopodium penninervium & Shrub & $\mathrm{x}$ & $\mathrm{x}$ & $\mathrm{x}$ \\
\hline Sterculiaceae & Dombeya torrida & Shrub & & & \\
\hline \multirow[t]{2}{*}{ Ulmaceae } & Celtis africana & Tree & $\mathrm{x}$ & & \\
\hline & Clerodendron myricoides & Tree & & $\mathrm{x}$ & \\
\hline \multirow[t]{2}{*}{ Verbenaceae } & Premna schimperi & Shrub & $\mathrm{x}$ & & $\mathrm{x}$ \\
\hline & Premna resinosa & Shrub & $\mathrm{x}$ & & \\
\hline Vitaceae & Vitis vinifera & Shrub & & & $\mathrm{x}$ \\
\hline
\end{tabular}

The study showed that an increasing trend in the total number of plant species from the early to the intermediate and mature stages following the natural sequence of successional stages that occurred subsequent to changes in land use system of the study area which is in line with the study reported for dry forests of Mexico [4].

The number of species that had been recorded in each of the successional stage ranges from 46-88 (Figures 1-3). The mature forest was found to have the highest species richness (88 species) followed by Intermediate stage (62 species) and Early stage of succession (46 species). The mature forest comprises $77.2 \%$ of the total number of species recorded in this study. The lowest number of species richness was recorded in ES1 sites (Figure 3) while the highest was in MS2 sites (Figure 1). There are bare lands in ES forest sites of this study and these could result in pronounced microclimatic changes and low water retention, which hinders the germination and establishment of typical of mature forests species as reported from elsewhere [4].

The number of species recorded in MS successional stage is almost twice more than the number of species recorded in ES succession. In this study, the number of species in MS sites was also higher than the IS sites which is not in line with the intermediate levels of disturbance (ILD) hypothesis $[9,6]$ but agreed with [5]. It had been reported from northern Chiloé Island [20] that the similarity in species between the successional stages predicts that the composition and community structure of plants change gradually, without total replacement of one community for another. Most Tropical Forest species may be found in all successional stages, but recruitment from MS could be lower in ES according to the initial floristic composition hypothesis [20] where each species had explicit environmental circumstances that limited its final richness and diversity. The number of species recorded in IS successional stage were equal to the total number species reported from Ades dry Afromontane forest [35].

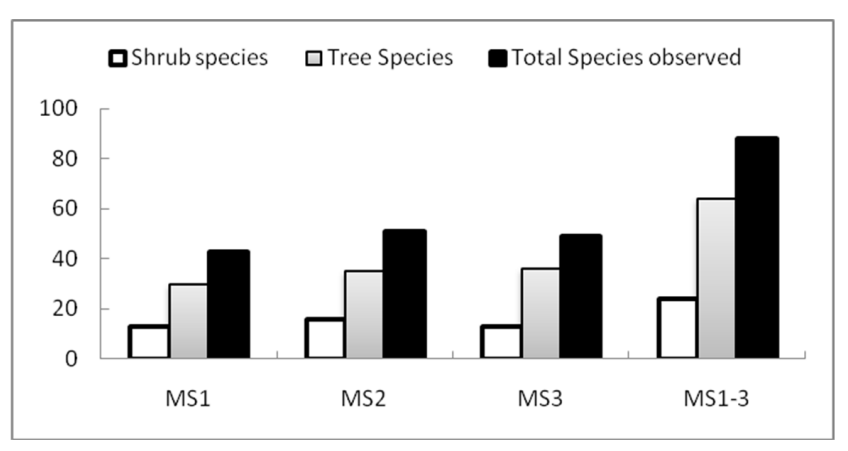

Figure 1. Number of Species observed in MS Sites.

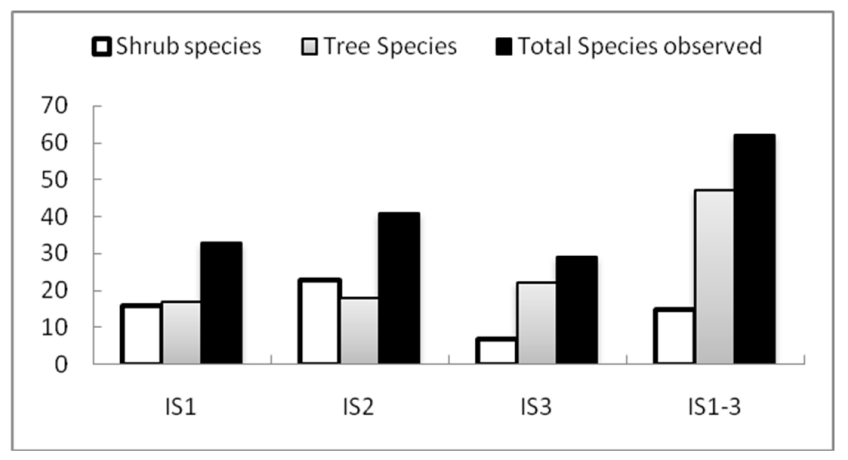

Figure 2. Number of Species observed in IS Sites.

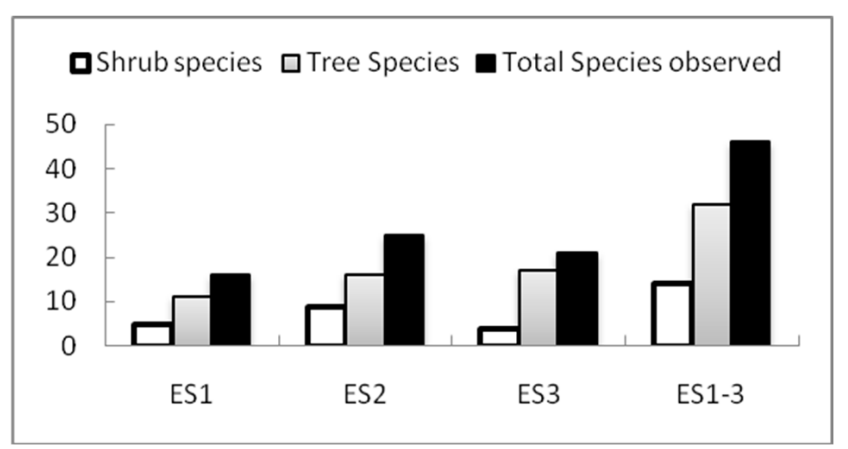

Figure 3. Number of Species observed in ES Sites. 
Analysis of Chao 2 estimator showed that $92.6 \%, 92.5 \%$ and $83.6 \%$ of the species respectively were present in the mature stage, in the intermediate stage, and in the early stage. The expected number of species recorded in each site ranged from $40 \%$ for ES1 sites to $96.1 \%$ for MS3 sites (Figure 4). This study revealed that an average of $89.6 \%$ of the total species expected had been found for each successional stage and the number species observed was close to the expected number of species richness taking only those woody species with $\mathrm{DBH}>10 \mathrm{~cm}$. It is comparable to similar study from tropical dry forest in southwest Mexico [4].

The total number of species rarefied in each successional site ranged from 12 for ES1 sites to 49 for MS2 sites (Figure 4). The overall total species rarefied were 37, 55 and 65 respectively for ES, IS and MS successional stages. The total number of species rarefied in ES1 sites in this study was by far lower than the 57 rarefied species reported for other forests [4].

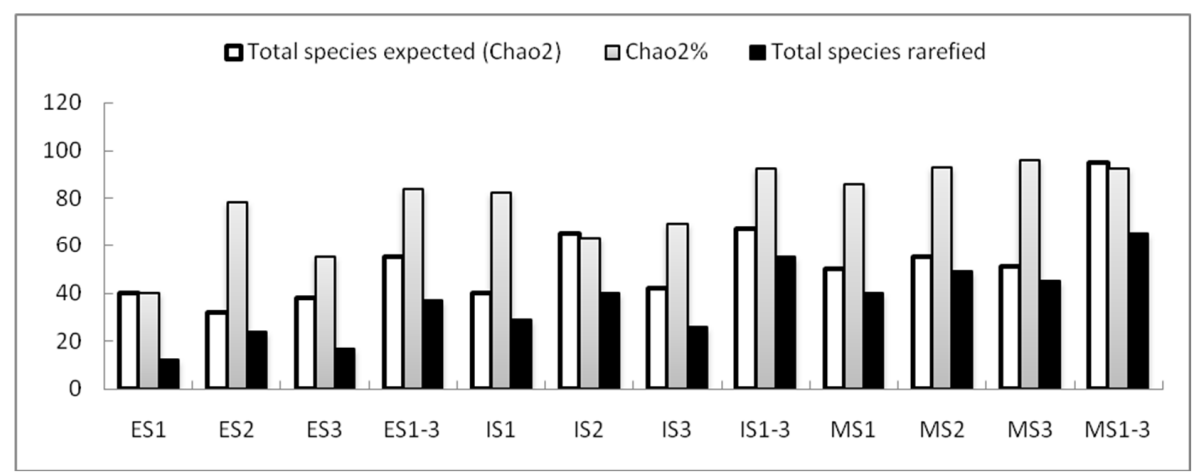

Figure 4. Expected and rarefied number of Woody plants for nine sites in 3 successional stages at Jello-Muktar dries afromontane forest, South-Eastern Ethiopia.

\subsection{Abundance of Trees and Shrubs}

In terms of the abundance of trees and shrubs, a total of 4990 individuals had been recorded. The highest and the lowest number of individuals were recorded in MS and ES succssional stages respectively (Figure 5). A total of 2330 individuals were recorded in MS sccessional stages comprising about $46.7 \%$ of the total individuals observed. With respect to the number of individuals in each site, it ranged from the lowest of 256 individuals for ES3 sites to the highest of 980 individuals for MS2 sites. The number of individuals recorded from the site of MS2 (980) was almost equal to the total number individuals observed in all ES sites (991). The number of individuals recorded in each successional stage of this study was higher than similar study reported for other dry tropical forests [4]. This could be due to geographic variation of the two forest types.

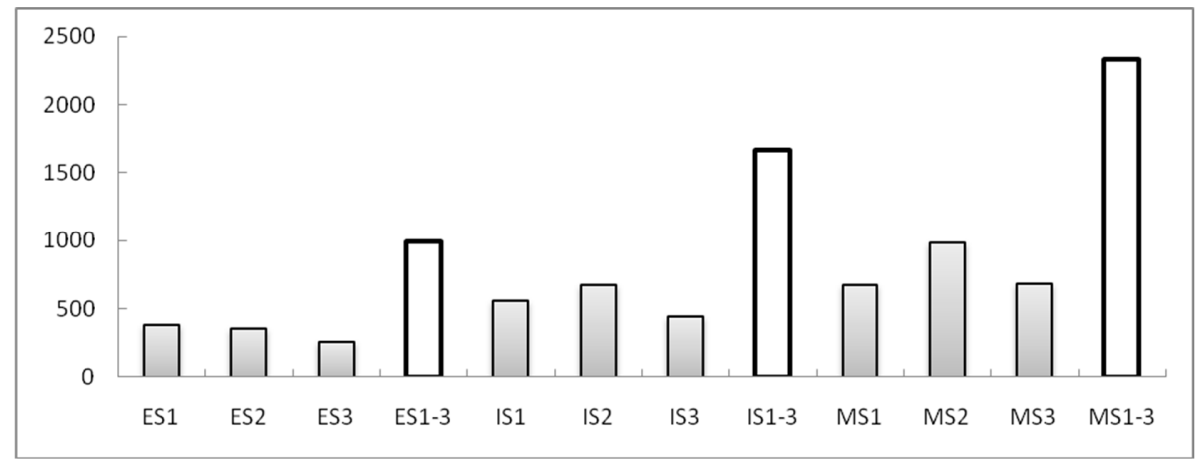

Figure 5. Abundance of Woody plants for nine sites (shaded bars) in 3 successional stages (Open bars) at Jello-Muktar dry afromontane forest, South-Eastern Ethiopia.

\subsection{Species Diversity}

Variation was also observed in Shannon index of diversity and Evenness indices between successional stages and among the sites. The index of Shannon-weiver ranged from 2.4 to 3.7. The highest mean Shannon-Wiener (3.7) was from MS2 sites (Figure 6) and the lowest was from sites of ES1 (2.4) indicating that MS had more species diversity than ES and IS sites. There was significant difference in H' among all the three sites of ES successional stages. Likewise sites of IS3 were significantly different from sites of IS1 and IS2 under IS succession. No significant variation was observed between MS1 and MS3 sites in MS succession. Overall there exist significant variation among ES, IS and MS successional stages (Figure 6). The overall mean Shannon-Wiener index of diversity for the three successional stages was 3.25 showing that the status of this forest under good diversity. The overall mean Shannon-Wiener index value recorded in this study was higher than the mean Shannon-Wiener Index of diversity of 2.82 reported for Ades dry afromontane forest of the same agro-ecology [35], Menagesha Suba Forest with $H^{\prime}=2.57$ [18] and Ylat forest with $H^{\prime}=2.94$ [44]. 


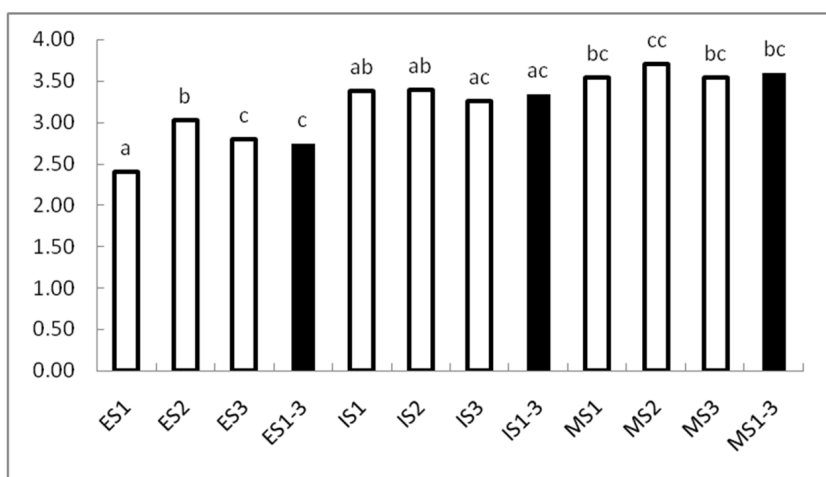

Figure 6. Shannon index of diversity for nine sites (Open bars) and three successional stages (shaded bars) of Jello-Muktar dry afromontane forest, South-Eastern Ethiopia. NB. Different letters denote significant differences $(p<0.05)$ according to independent $t$-test.

\subsection{Species Evenness}

Evenness values also vary between succsseional stages (Figure 7). The lowest Evenness value of 0.67 was from sites of ES1 in ES successional stages. Sites of ES1 were significantly different from ES2 and ES3 sites of ES successional stage. In the IS successional stage, sites of IS3 were significantly different from IS1 and IS2 sites. With respect to MS successional stages, MS1 were significantly different from sites of MS2 and MS3. Comparison of Evenness values among sites from different successional stages showed that no significant differences was observed among sites of ES2, ES3, IS1 and IS2 in ES and IS successional stages on the one hand IS3, MS2 and MS3 from IS and MS successional stages on the other. The overall mean measure of evenness in this study was about 0.86 . This means that the relative homogeneity of the species in the samples was $86 \%$ of the maximum possible even population. The lower Evenness in the ES1 sites shows a typical pattern of disturbed communities in which pioneer species achieve high relative importance values in relation to other species as suggested $[37,6]$.

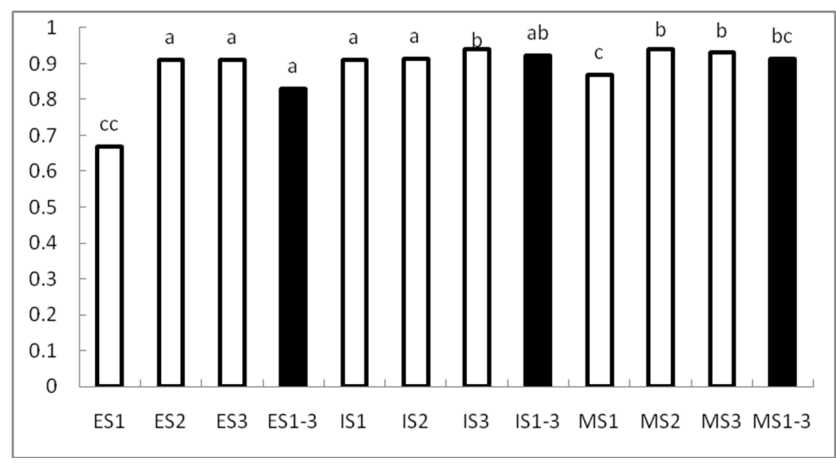

Figure 7. Evenness indices from nine sites (open bars) and three successional stages at Jello-Muktar dry afromontane forest, South-Eastern Ethiopia. NB. Different letters denote significant differences $(p<0.05)$ according to independent $t$-test.

\section{Conclusions and Recommendations}

A total of 114 species (76 tree and 38 shrub species) corresponding to 51 families were recorded in the three successional stages. The number of species that had been recorded in each of the successional stage ranges from 46-88. The mature forest was found to have the highest species richness (88 species) followed by Intermediate stage (62 species) and Early stage of succession (46 species). The mature forest comprises $77.2 \%$ of the total number of species recorded in this study. Variation was also observed in Shannon index of diversity and Evenness indices between successional stages and among the sites. The study showed that an increasing trend in the total number of plant species from the early to the intermediate and mature stages following the natural sequence of successional stages that occurred subsequent to changes in land use system of the study area.

Strategies and conservation priorities should be aimed at maintaining different successional plant communities with heterogeneous physiognomy in addition to mature forests in order to sustain regeneration processes and a high level of plant diversity.

\section{Acknowledgements}

This work was funded by the office of the Research and Extension Affaires of Haramaya University. We are grateful to Oda Bultum University for facilitation of the whole work. Thanks also go to The Research and Community Engagement Directorate of Oda Bultum University for its continuous financial facilitation, follow-up and comments during the research work. We thank Mr. Husen Yusuf for assisting with field work and for providing background information about the study site. Thanks also go to Oromia Forest and Wildlife enterprise of Hararghe branch for providing field equipments and basic information regarding the current and past history of the forest. Thanks also go to Key informants and development agents particularly $\mathrm{Mr}$. Mekonnen Worku for his guidance during field data collection.

\section{References}

[1] Abate Ayalew, Tamrat Bakele, Sebsebe Demissew. 2006. The undifferentiated fromontane Forest of Denkoro in the Central Highland of Ethiopia: Floristic and Structural Analysis. SINET. Journal of Science. 29: 45-56.

[2] Abera A. and Yasin A. 2018. Diversity and Abundance of Woody Plant Species of Assosa Forest Field Gene, Benishanigul Gumuz Regional State, Western Ethiopia. International Journal of Plant Biology \& Research 6 (5): 1100.

[3] Aide, T. M., J. K. Zimmerman, J. B. Pascarella, L. Rivera and H. Marcano-Vega. 2000. Forest regeneration in a chronosequence of tropical abandoned pastures: implications for restoration ecology. Restoration Ecology 8: 328-338.

[4] Almazán-Núñez, María del Coro Arizmendi, Luis E. Eguiarte and PabloCorcuera. 2012. Changes in composition, diversity and structure of woody plants in successional stages of tropical dry forest in southwest Mexico. Revista Mexicana de Biodiversidad 83: 1096-1109. DOI: 10.7550/rmb.30403. 
[5] Aravena, J. C., M. R. Carmona, C. A. Pérez and J. J. Armesto. 2002. Changes in tree species richness, stand structure and soil properties in a successional chronosequence in northern Chiloé, Chile. Revista Chilena de Historia Natural 75: 339-360.

[6] Bongers, F., L. Poorter, W. D. Hawthorne and D. Sheil. 2009. The intermediate disturbance hypothesis applies to tropical forests, but disturbance contributes little to tree diversity. Ecology Letters 12: 1-8.

[7] Ceccon, E., P. Huante and E. Rincón. 2006. Abiotic factors influencing tropical dry forests regeneration. Brazilian Archives of Biology and Technology 49: 305-312.

[8] Chapman, C. A. and L. J. Chapman. 1990. Density and growth rate of some tropical dry forest trees: Comparisons between successional forest types. Bulletin of the Torrey Botanical Society 117: 226-231.

[9] Collins, S. L. and S. M. Glenn. 1997. Intermediate disturbance and its relationship to within- and between-patch dynamics. New Zealand Journal of Ecology 21: 103-110.

[10] Collins, S. L., S. M. Glenn and D. J. Gibson. 1995. Experimental analysis of intermediate disturbance and initial floristic composition: decoupling cause and effect. Ecology 76: 486-492.

[11] Colwell, R. K. 2006. EstimateS: statistical estimation of species richness and shared species from samples. Version 8. URL: http//viceroy.eeb.uconn.edu/estimates

[12] Colwell, R. K. and J. A. Coddington. 1994. Estimating terrestrial biodiversity. Philosophical Transactions of the Royal Society of London Series B 345: 101-118.

[13] Connell, J. H. 1978. Diversity in tropical rain forests and coral reefs. Science 199: 1302-1310. Royal Society of London Series B 345: 101-118.

[14] Connell, J. H and R. O. Slatyer. 1977. Mechanisms of succession in natural communities and their role in community stability and organization. American Naturalist 111: 1119-1.

[15] Cristian Echeverría Adrian C. Newton, Antonio Lara, José María Rey Benayas and David A. Coomes. 2007. Impacts of forest fragmentation on species composition and forest structure in the temperate landscape of southern Chile. Global Ecology and Biogeography.

[16] Davies, J. S. and H. Semui. 2006. Competitive dominance in a secondary successional rain-forest community in Borneo. Journal of Tropical Ecology 22: 53-64.

[17] DeClerck, F. A. J., R. Chazdon, K. D. Holl, J. C. Milder, B. Finegan, A. Martínez-Salinas, P. Imbach, L. Canet and Z. Ramos. 2010. Biodiversity conservation in human modified landscapes of Mesoamerica: Past, present and future. Biological Conservation 143: 2301-2313.

[18] Dinkissa Beche. 2011. Floristic Composition, Diversity and Structure of Woody Plant Species in Menagesha Suba State Forest, Central Ethiopia. M. Sc. Thesis Addis Ababa University, Addis Ababa.

[19] EMA (Ethiopian Mapping Agency). 1999. Bedessa (Kuni), Ethiopian Topo Sheet 1:50,000. ETH-4, 0840 B2, EMA, Addis Ababa, Ethiopia.

[20] Egler, F. E. 1954. Vegetation science concepts I. Initial floristic composition - a factor in old-field vegetation development. Vegetation 4: 412-417.
[21] Eyelachewu Zewdie. 1999. Selected Physical, Chemical and Mineralogical Characteristics of Major Soils Occurring in Chercher Highlands, Eastern Ethiopia. Ethiopian Journal of Natural Resources Vol. 1, No 2, pp 173-185.

[22] Gentry, A. H. 1995. Diversity and floristic composition of neotropical dry forests. In Seasonally dry tropical forests, S. H. Bullock, H. A. Mooney and E. Medina. (eds.). Cambridge University Press, Cambridge. p. 146-194.

[23] Gillespie, T. W., A. Grijalva and C. N. Farris. 2000. Diversity, composition, and structure of tropical dry forests in Central America. Plant Ecology 147: 37-47.

[24] Griscom, H. P. and M. S. Ashton. 2011. Restoration of dry tropical forests in Central America: A review of pattern and process. Forest Ecology and Management 161: 1564-1579.

[25] Janzen, D. H. 1988b. Tropical dry forests: the most endangered major ecosystem. In Biodiversity, E. O. Wilson (ed.). National Academic Press. Washington. p. 130-137.

[26] Jha, C. S. and S. J. Singh. 1990. Composition and dynamics of dry tropical forest in relation to soil texture. Journal of Vegetation Science 1: 609-614.

[27] Kayes, L. J., P. D. Anderson and K. J. Puettmann. 2010. Vegetation succession among and within structural layers following wildfire in managed forests. Journal of Vegetation Science 21: 233-247.

[28] Killeen, T. J. A. Jardim, F. Mamani and N. Rojas. 1998. Diversity, composition and structure of a tropical semi deciduous forest in the Chiquitanía region of Santa Cruz, Bolivia. Journal of Tropical Ecology 14: 803-827.

[29] Kitessa Hundera and Tsegaye Gadissa. 2008. Vegetation Composition and Structure of Belete Forest, Jimma Zone, South Western Ethiopia. Ethiopia. Journal of Biological Sciences 7 (1): 1-15.

[30] Lulekal, E., E. Kelbessa, T. Bekele\& H. Yineger. 2008. An ethnobotanical study of medicinal plants in ManaAngetu District, southeastern Ethiopia. Journal of Ethnobiology and Ethnomedicine 4: 10. dx.doi.org/10.1186/1746-42694-10.

[31] Madeira, B. G., M. M. Espírito-Santo, S. D. Neto, Y. R. F. Nunes, G. A. Sánchez-Azofeita, G. Wilson and Melese Bekele, 2016. Floristic Composition and Vegetation Structure of Woody Species in Lammo Natural Forest in Tembaro Woreda, Kambata-Tambaro Zone, Southern Ethiopia. American Journal of Agriculture and Forestry. Vol. 4, No. 2, pp. 49-55. doi: 10.11648/j.ajaf.20160402.16.

[32] Mesfin Tadesse \& Lisanework Nigatu. 1996. An ecological and ethnobotanical study of wild or spontaneous coffee, Coffea arabica in Ethiopia. In: L. J. G. van der Maesen, X. M. van der Burgt, J. M. van Medenbach deRooy (eds.), The Biodiversity of African Plants. Proceedings XIVth AETFAT Congress, 22-27 August 1994, Wageningen, The Netherlands, Kluwer Academic Publishers. Pp 277-294.

[33] MOA/FAO/UNDP. 1983. Generalized Agroclimatic Map, Ethiopia. Assistance to Land Use Planning Project. FAO/UNDP-ETH/78/003. Addis Ababa. Ethiopia.

[34] Mohammed Assen, P. A. L. le Roux, C. H. Barker, and Heluf Gebrekidan. 2005. Soils of Jelo Micro- Catchment in the Chercher Highlands of Eastern Ethiopia: I Morphological and Physicochemical Properties. Ethiopian Journal of Natural Resources, 7 (1): 55-81. 
[35] Muktar Reshad, Alemayehu Beyene, Muktar Mohammed. Woody Species Richness and Diversity at Ades Dry Afromontane Forest of SouthEastern Ethiopia. American Journal of Agriculture and Forestry. Vol. 7, No. 2, 2019, pp. 44-52. doi: 10.11648/j.ajaf.20190702.12.

[36] Murphy, P. G. and A. E. Lugo. 1986. Ecology of tropical dry forest. Annual Review of Ecology and Systematic 17: 67-88.

[37] Onaindia, M., I. Dominguez, I. Albizu, C. Garbisu and I. Amezaga. 2004. Vegetation diversity and vertical structure as indicators of forest disturbance. Forest Ecology and Management 195: 341-354.

[38] Powers, J. S., J. M. Becknell, J. Irving and D. Pèrez-Aviles. 2009. Diversity and structure of regenerating tropical dry forests in Costa Rica: Geographic patterns and environmental drivers. Forest Ecology and Management 258: 959-970.

[39] Roxburgh, S. H., K. Shea and J. Bastow. 2004. The intermediate disturbance hypothesis: patch dynamics and mechanisms of species coexistence. Ecology 85: 359-371.

[40] Sabogal, C. 1992. Regeneration of tropical dry forests in central America, with examples from Nicaragua. Journal of Vegetation Science 3: 407-416.

[41] Sanders, HL. 1968. Marine benthic diversity: a comparative study. American Naturalist 102: 243-282.
[42] Simon Shibru and Girma Balcha. 2004. Composition, Structure and regeneration status of woody species in Dindin Natural Forest, Southeast Ethiopia: An implication for conservation. Ethiopian Journal of Biological Science. (1) 3: 15-35.

[43] Shambel Bantiwalu. 2010. Floristic Composition, Structure and Regeneration Status of Plant Species in Sanka Meda Forest, Guna District, Arsi zone of Oromiya Region, Southeast Ethiopia.

[44] Sisay. 2016. Vegetative structure, floristic composition and natural regeneration of a species in ylat forest in meket woreda, north eastern Ethiopia.

[45] Tamrat Bekele. 1993. Vegetation and Ecology of Afromontane Forests on the Central Plateau of Shewa, Ethiopia. Acta Phytogeogr. Suec. 79, 64 Pp.

[46] Tansley, A. G. 1935. The use and abuse of vegetational concepts and terms. Ecology 16: 284-307.

[47] Vieira, D. L. M. and A. Scariot. 2006. Principles of natural regeneration of tropical dry forests for restoration. Restoration Ecology 14: 11-20. 